International Journal of Pure and Applied Mathematics

Volume 108 No. 2 2016, 451-466

ISSN: 1311-8080 (printed version); ISSN: 1314-3395 (on-line version)

url: http://www.ijpam.eu

doi: 10.12732/ijpam.v108i2.18

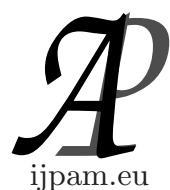

\title{
ON PLANARITY OF 3-JUMP GRAPHS
}

\author{
Varanoot Khemmani ${ }^{1}$, Chira Lumduanhom ${ }^{2 \S}$, \\ Sriwan Muangloy ${ }^{3}$, Massiri Muanphet ${ }^{4}$, Kittisak Tipnuch ${ }^{5}$ \\ $1,2,3,4,5$ Department of Mathematics \\ Srinakharinwirot University \\ Sukhumvit 23, Bangkok, 10110, THAILAND
}

\begin{abstract}
For a graph $G$ of size $m \geq 1$ and edge-induced subgraphs $F$ and $H$ of size $k$ where $1 \leq k \leq m$, the subgraph $H$ is said to be obtained from the subgraph $F$ by an edge jump if there exist four distinct vertices $u, v, w$ and $x$ such that $u v \in E(F), w x \in E(G)-E(F)$, and $H=F-u v+w x$. The $k$-jump graph $J_{k}(G)$ is that graph whose vertices correspond to the edge-induced subgraphs of size $k$ of $G$ where two vertices $F$ and $H$ of $J_{k}(G)$ are adjacent if and only if $H$ can be obtained from $F$ by an edge jump.
\end{abstract}

All connected graphs $G$ for whose $J_{3}(G)$ is planar are determined.

AMS Subject Classification: $05 \mathrm{C} 10,05 \mathrm{C} 12$

Key Words: $k$-jump distance, $k$-jump graph, 3 -jump graph, planar graph

\section{Introduction}

The concept of the $k$-jump graph of a nonempty graph $G$ of size $m$ where $1 \leq k \leq m$ was introduced by Chartrand, Hevia, Jarrett and Schultz [1]. Let $G$ be a graph of size $m \geq 1$ and $F$ and $H$ be edge-induced subgraphs of size $k$ of $G$ where $1 \leq k \leq m$. The subgraph $H$ is said to be obtained from the subgraph $F$ by an edge jump if there exist four distinct vertices $u, v, w$ and $x$ such that $u v \in E(F), w x \in E(G)-E(F)$, and $H=F-u v+w x$. It is obvious that if $H$ is obtained from $F$ by an edge jump then $F$ is obtained from

Received: January 27, 2016

Published: June 23, 2016

${ }^{\S}$ Correspondence author (c) 2016 Academic Publications, Ltd.

url: www.acadpubl.eu 
$H$ by an edge jump. If there is a sequence $F=H_{0}, H_{1}, \ldots, H_{\ell}=H$ where $\ell \geq 1$ of edge-induced subgraphs of size $k$ such that $H_{i+1}$ is obtained from $H_{i}$ by an edge jump for $0 \leq i \leq \ell-1$, then we say that $F$ can be $j$-transformed into $H$. The minimum number of edge jumps required to $j$-transform $F$ into $H$ is the $k$-jump distance from $F$ to $H$. For a graph $G$ of size $m \geq 1$ and an integer $k$ with $1 \leq k \leq m$, the $k$-jump graph $J_{k}(G)$ is that graph whose vertices correspond to the edge-induced subgraphs of size $k$ of $G$ where two vertices $F$ and $H$ of $J_{k}(G)$ are adjacent if and only if the $k$-jump distance between edge-induced subgraphs $F$ and $H$ is 1 that is, $H$ is obtained from $F$ by an edge jump. We can label each vertex of $J_{k}(G)$ by listing all the edges of the respective subgraph. The concept of the $k$-jump graph is illustrated in Figure 1. In particular, if $k=1$ then the graph $J_{1}(G)=J(G)$ is called the jump graph of $G$. Moreover, $J(G)=\overline{L(G)}$, the complement of the line graph of $G$. In [3] all connected graphs $G$ for whose $J_{2}(G)$ is planar are determined in terms of a finite set $S$ of graphs, namely a connected graph $G$ has a planar 2-jump graph if and only if $G$ is a subgraph of some element of $S$. The goal of this paper is to characterize all connected graphs having a planar 3-jump graph along the same lines as the characterization of connected graphs having a planar 2-jump graph.

$G$ :
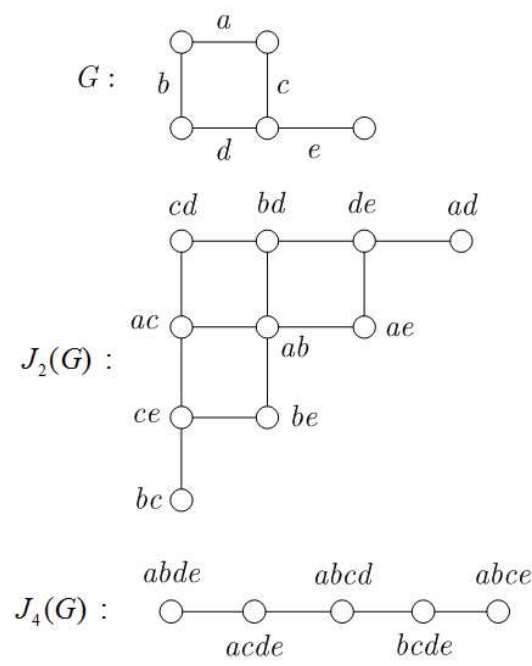

$$
J_{1}(G)=\overline{L(G)}
$$
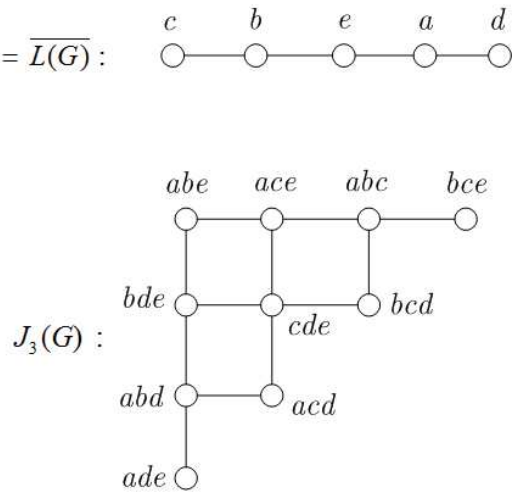

$J_{5}(G)$ :

abcde

Figure 1: The $k$-jump graphs of a graph

The following results appeared in [3] and [4] will be useful for us later.

Theorem 1.1. ([4]) A graph is planar if and only if it contains no 
subgraph isomorphic to $K_{5}$ or $K_{3,3}$ or a subdivision of one of these graphs.

Theorem 1.2. ([3]) If $G$ is a graph of size $m \geq 1$ and $k$ is an integer with $1 \leq k<m$, then $J_{k}(G)=J_{m-k}(G)$.

Theorem 1.3. ([3]) If a graph $G$ is a subdivision of a graph $H$ and $J_{k}(H)$ is nonplanar for some positive integer $k$, then $J_{k}(G)$ is nonplanar.

The reader is referred to the book [2] by Chartrand, Lesniak and Zhang for basic definitions and terminology not described here.

\section{Connected Graphs with Planar 3-Jump Graphs}

In this section we will focus our attention on the planarity of the 3-jump graph $J_{3}(G)$ for a connected graph $G$ of size at least 3 . As we mentioned earlier, our aim is to determine a finite set $S$ of graphs with the property that a connected graph $G$ has a planar 3-jump graph if and only if $G$ is a subgraph of some element of $S$.

In [3] it is shown that for the path $P_{n}$ and cycle $C_{n}$ of order $n, J_{2}\left(P_{n}\right)$ is nonplanar if and only if $n \geq 6$ and $J_{2}\left(C_{n}\right)$ is nonplanar if and only if $n \geq 5$. Similar results can be obtained for $J_{3}\left(P_{n}\right)$ and $J_{3}\left(C_{n}\right)$. The 3 -jump graphs $J_{3}\left(P_{5}\right), J_{3}\left(P_{6}\right), J_{3}\left(C_{4}\right)$, and $J_{3}\left(C_{5}\right)$ are shown in Figure 2 which we see that $J_{3}\left(P_{5}\right)$ and $J_{3}\left(C_{4}\right)$ are planar while $J_{3}\left(P_{6}\right)$ and $J_{3}\left(C_{5}\right)$ are nonplanar. Therefore, by Theorems 1.1 and 1.3 , the following results are immediate.

Corollary 2.1. For $n \geq 4, J_{3}\left(P_{n}\right)$ is nonplanar if and only if $n \geq 6$.

Corollary 2.2. For $n \geq 3, J_{3}\left(C_{n}\right)$ is nonplanar if and only if $n \geq 5$.

We now present a simple but useful lemma.

Lemma 2.3. If $H$ is a subgraph of a connected graph $G$ then, for each $k, J_{k}(H)$ is a subgraph of $J_{k}(G)$.

Proof. Let $k$ be an integer such that $1 \leq k \leq m$ where $m$ is the size of $G$. If $v_{H_{1}}$ is a vertex of $J_{k}(H)$ that corresponds with an edge-induced subgraph $H_{1}$ of size $k$ of $H$ then since $H$ is a subgraph of $G, H_{1}$ is certainly an edge-induced subgraph of size $k$ of $G$. Thus $v_{H_{1}}$ is a vertex of $J_{k}(G)$ and so $V\left(J_{k}(H)\right) \subseteq$ $V\left(J_{k}(G)\right)$. On the other hand, if $e=v_{H_{1}} v_{H_{2}} \in E\left(J_{k}(H)\right)$ then in a graph $H$, $H_{1}$ is obtained from $H_{2}$ by an edge jump. Now, since $H$ is a subgraph of $G$, 


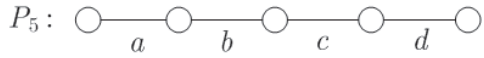

$P_{6}:$
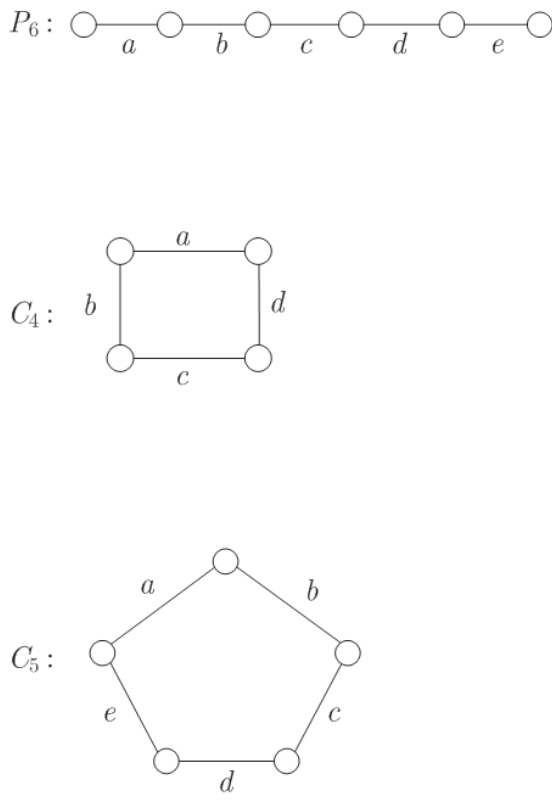
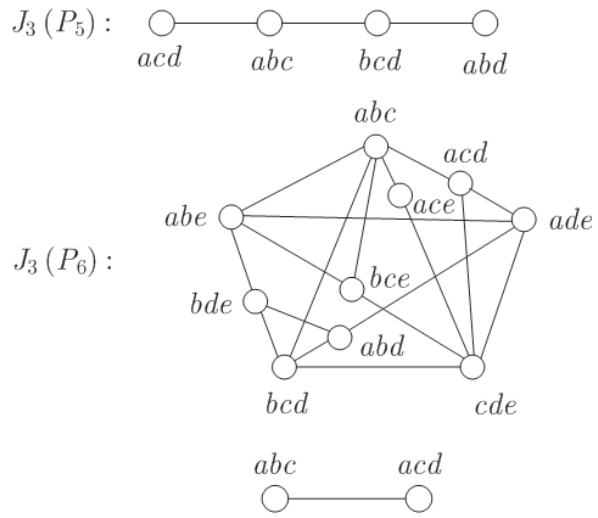

$J_{3}\left(C_{4}\right):$
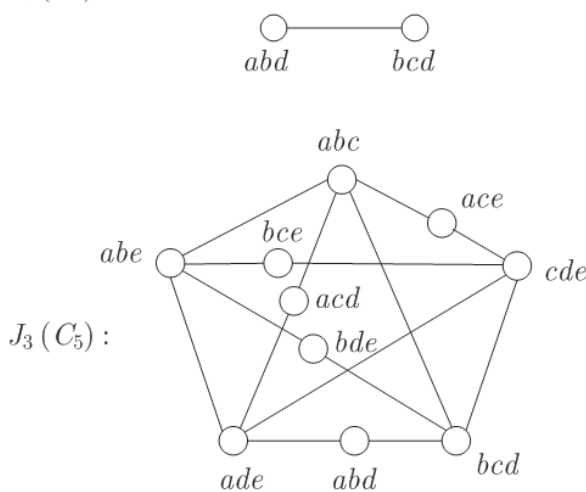

Figure 2: The 3-jump graphs of $P_{5}, P_{6}, C_{4}$ and $C_{5}$

it follows that in $G, H_{1}$ is also obtained from $H_{2}$ by an edge jump. Therefore $e=v_{H_{1}} v_{H_{2}} \in E\left(J_{k}(G)\right)$ and so $E\left(J_{k}(H)\right) \subseteq E\left(J_{k}(G)\right)$.

Theorem 1.1 and lemma 2.3 give us the following results.

Lemma 2.4. If $G$ is a graph containing a subgraph $H$ where $H$ is the union of edge-disjoint subgraphs $H_{1}$ of size at least 2 and $H_{2}$ of size at least 4 such that (1) $H_{1}$ contains edges $b$ and $e$, (2) $H_{2}$ contains edges $a, c, d$ and $f$ where $a$ is not adjacent to $c$, and (3) edges $b$ and $e$ are not adjacent to both $d$ and $f$ in $H$, then $J_{3}(G)$ is nonplanar.

Proof. Since there exists a subgraph of the 3 -jump graph $J_{3}(H)$ that is isomorphic to a subdivision of $K_{3,3}$ as shown in Figure 3, it follows that $J_{3}(H)$ and so $J_{3}(G)$ are nonplanar. 


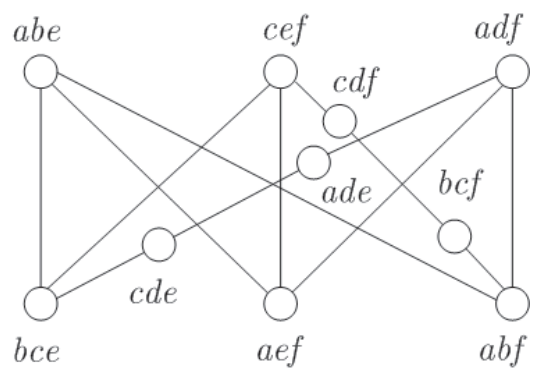

Figure 3: A subgraph of $J_{3}(H)$ of Lemma 2.4

Lemma 2.5. If $G$ is a graph containing a subgraph $H$ where $H$ is the union of edge-disjoint subgraphs $H_{1}$ of size at least 2 and $H_{2}$ of size at least 4 such that (1) $H_{1}$ contains nonadjacent edges $a$ and $f$, (2) $H_{2}$ contains edges $b$, $c, d$ and $e$ where $b$ and $d$ are not adjacent, (3) $a$ is not adjacent to $c$ in $H$, and (4) $f$ is not adjacent to two edges $b$ and $e$ in $H$, then $J_{3}(G)$ is nonplanar.

Proof. Since the 3-jump graph $J_{3}(H)$ contains a subgraph isomorphic to a subdivision of $K_{3,3}$ as shown in Figure 4, it follows that $J_{3}(H)$ and thus $J_{3}(G)$ are nonplanar.

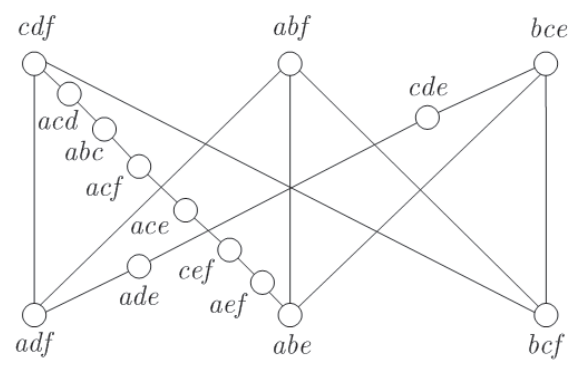

Figure 4: A subgraph of $J_{3}(H)$ of Lemma 2.5

Lemma 2.6. If $G$ is a graph containing three subgraphs $G_{1}, G_{2}$, and $G_{3}$ where $G_{1}$ is isomorphic to $K_{1,3}$ or $K_{3}$, and $G_{2}$ and $G_{3}$ are both isomorphic to $P_{2}$ such that for each $i \in\{2,3\}, G_{1}$ and $G_{i}$ are disjoint subgraphs, and $G_{2}$ and $G_{3}$ are edge-disjoint subgraphs, then $J_{3}(G)$ is nonplanar.

Proof. If the edge sets of three subgraphs $G_{1}, G_{2}$, and $G_{3}$ of $G$ are $E\left(G_{1}\right)=$ $\{e, f, g\}, E\left(G_{2}\right)=\{a\}$, and $E\left(G_{3}\right)=\{d\}$ respectively, then $G$ contains either 
$H_{1}, H_{2}, H_{3}$ or $H_{4}$, shown in Figure 5, as a subgraph. Consequently, $J_{3}(G)$ contains a subgraph isomorphic to a subdivision of $K_{3,3}$ as shown in Figure 6. Thus $J_{3}(G)$ is nonplanar.
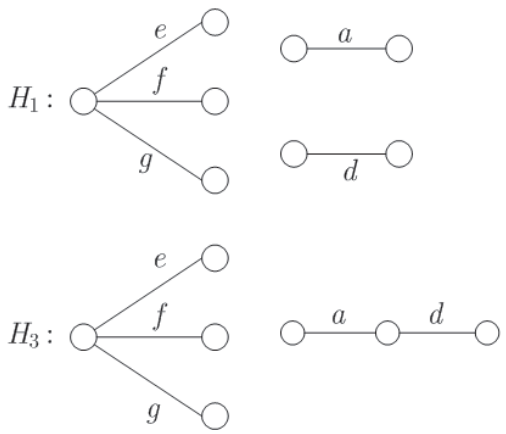

$H_{4}:$

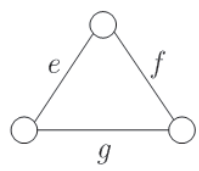

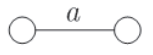
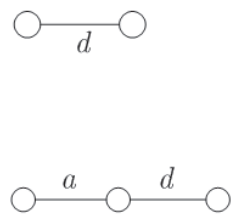

Figure 5: The four possible subgraphs for a graph $G$ of Lemma 2.6

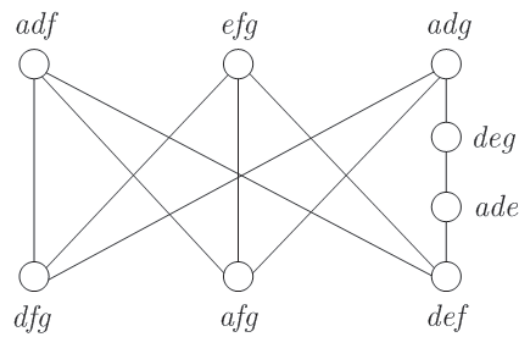

Figure 6: A subgraph of $J_{3}(G)$ of Lemma 2.6

We are next interested in showing that each of the graphs $N_{i}$ for $1 \leq i \leq 20$ of Figure 7 and each of the graphs $M_{i}$ for $1 \leq i \leq 11$ of Figure 10 have nonplanar and planar 3-jump graphs, respectively.

Theorem 2.7. For each graph $N_{i}$, where $1 \leq i \leq 20$, of Figure $7, J_{3}\left(N_{i}\right)$ is nonplanar.

Proof. We have seen in Corollaries 2.1 and 2.2 that $J_{3}\left(P_{6}\right)$ and $J_{3}\left(C_{5}\right)$ are nonplanar. Thus it remains to show the nonplanarity for $J_{3}\left(N_{i}\right)$ where $1 \leq i \leq 18$.

For $i \in\{1,5,11,16,18\}$, some subgraph of $J_{3}\left(N_{i}\right)$ is shown in Figure 8(a), $(b),(c),(d)$ and $(e)$, respectively. Since, for each $i, J_{3}\left(N_{i}\right)$ contains a subgraph 
that is isomorphic to either a subdivision of $K_{5}$ or a subdivision of $K_{3,3}, J_{3}\left(N_{i}\right)$ is nonplanar.

For $i \in\{2,3,4,7,8,12,13,15,17\}, N_{i}$ contains subgraphs $G_{1}, G_{2}$ and $G_{3}$ as mentioned in Lemma 2.6. (all edges of $N_{i}$ are labeled to be corresponding with the edges of $G_{1}, G_{2}$ and $G_{3}$ in Lemma 2.6.) Thus, by Lemma 2.6, it follows that $J_{3}\left(N_{i}\right)$ is nonplanar.

For $i \in\{6,9\}, N_{i}$ contains a subgraph $H$ as mentioned in Lemma 2.4. (all edges of $N_{i}$ are labeled to be corresponding with the edges of $H_{1}$ and $H_{2}$ in Lemma 2.4.) Thus, by Lemma 2.4, $J_{3}\left(N_{i}\right)$ is nonplanar.

For $i \in\{10,14\}, N_{i}$ contains a subgraph $H$ as mentioned in Lemma 2.5. (all edges of $N_{i}$ are labeled to be corresponding with the edges of $H_{1}$ and $H_{2}$ in Lemma 2.5.) Thus, by Lemma $2.5, J_{3}\left(N_{i}\right)$ is nonplanar.

An alternative way to show that $J_{3}\left(N_{i}\right)$ where $i \in\{1,2, \ldots, 20\}-\{10,13,14\}$ is nonplanar can be obtained from the following theorem.

Theorem 2.8. If $G$ is a connected graph of size $m$ and $H$ is a subgraph of $G$ of size $m$ ' such that $J_{k}(H)$ is nonplanar then $J_{k+m-m^{\prime}}(G)$ is nonplanar.

Proof. If $H=G$ then the result is trivial. Assume that $H$ is a proper subgraph of $G$ and so $m-m^{\prime} \geq 1$. We show that $J_{k+m-m^{\prime}}(G)$ contains a subgraph $F$ isomorphic to $J_{k}(H)$ which is nonplanar. Let $e_{1}, e_{2}, \ldots, e_{m-m^{\prime}} \in E(G)-E(H)$. Now, for each vertex $X$ of $J_{k}(H)$, let $e_{1} e_{2} \ldots e_{m-m^{\prime}} X$ be a vertex of $F$. Thus $V(F) \subseteq V\left(J_{k+m-m^{\prime}}(G)\right)$. Since $e_{1} e_{2} \ldots e_{m-m^{\prime}} X$ and $e_{1} e_{2} \ldots e_{m-m^{\prime}} Y$ are adjacent in $F$ if and only if $X$ and $Y$ are adjacent in $J_{k}(H)$, it follows that $F$ is isomorphic to $J_{k}(H)$.

In [3] it has been shown that for each graph $H_{i}$ where $1 \leq i \leq 17$ in Figure 9, $J_{2}\left(H_{i}\right)$ is nonplanar. Observe that $H_{15}=N_{1}, H_{14}=N_{2}, H_{9}=N_{3}, H_{13} \subsetneq N_{4}$, $H_{10}=N_{5}, H_{11}=N_{6}, H_{12}=N_{7}, H_{1} \subsetneq N_{8}, H_{2}=N_{9}, H_{1} \subsetneq N_{11}, H_{7}=N_{12}$, $H_{4}=N_{15}, H_{5} \subsetneq N_{16}, H_{5} \subsetneq N_{17}, H_{3} \subsetneq N_{18}, H_{17}=N_{19}$ and $H_{16}=N_{20}$. Thus by theorem $2.8, J_{3}\left(N_{i}\right)$ is nonplanar.

We now turn our attention to graphs having a planar 3-jump graph. If $G=K_{1, m}$ is a star of size $m$ then $J_{k}(G)$ is an empty graph of order $\left(\begin{array}{c}m \\ k\end{array}\right)$. Thus we have an immediate result.

Proposition 2.9. If $G$ is a star of size $m$ then $J_{k}(G)$ is planar for every $k$ where $1 \leq k \leq m$. 

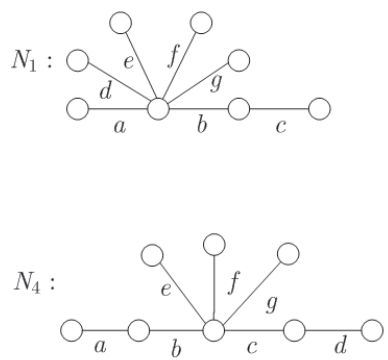

$N_{5}:$
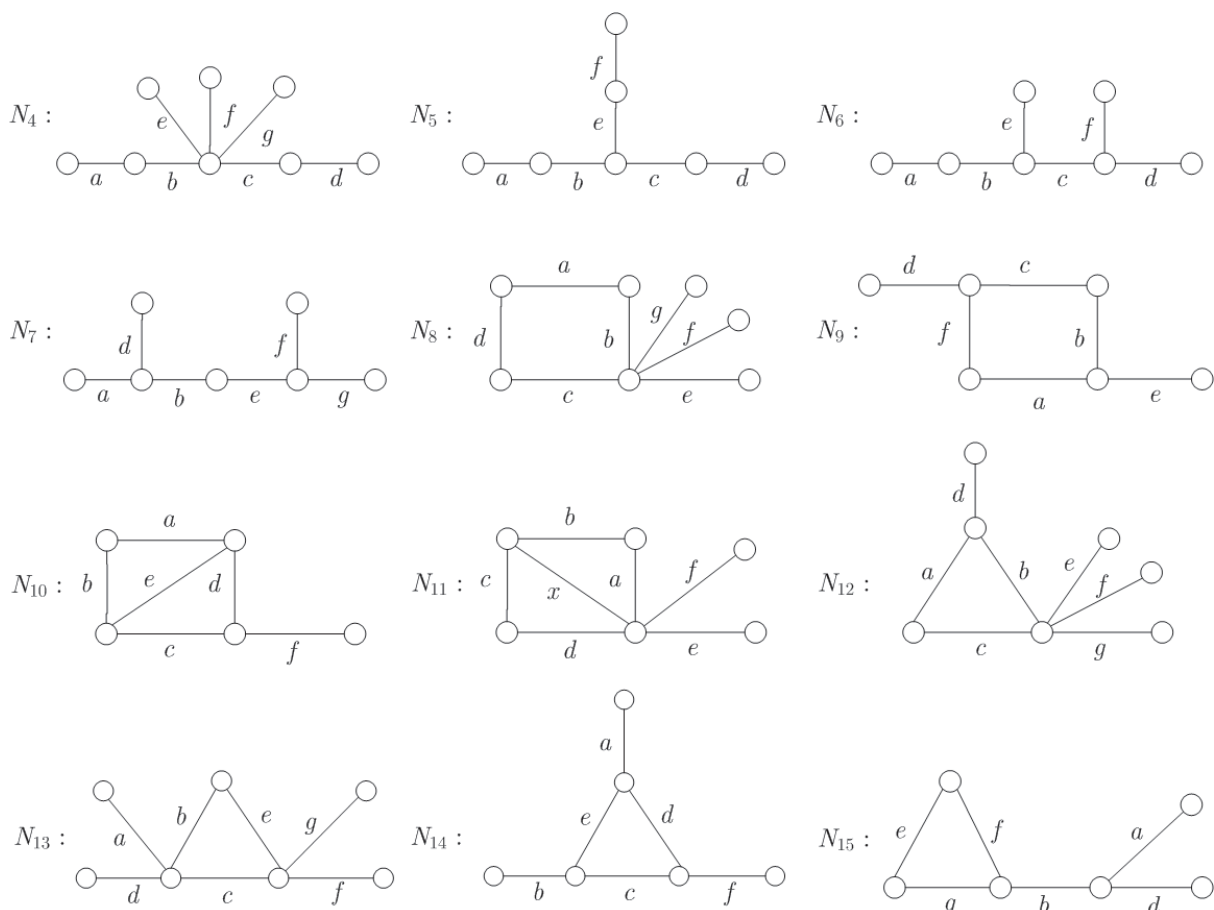

$N_{14}:$
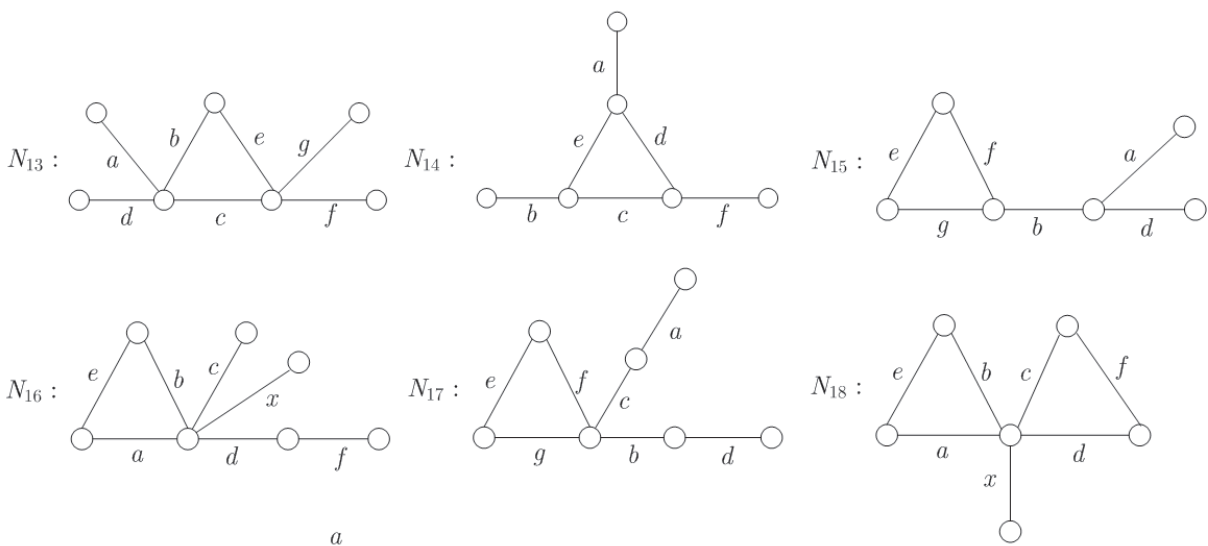

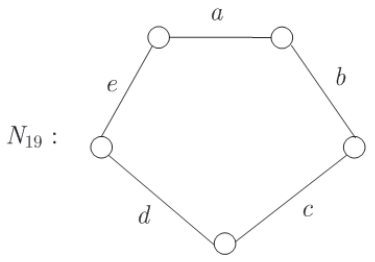

$N_{20}$ :

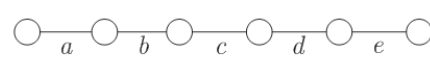

Figure 7: Graphs having a nonplanar 3-jump graph

Lemma 2.10. For each graph $M_{i}$, where $1 \leq i \leq 11$, of Figure $10, J_{3}\left(M_{i}\right)$ 


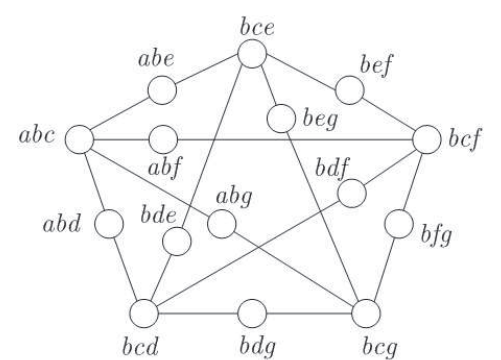

(a)

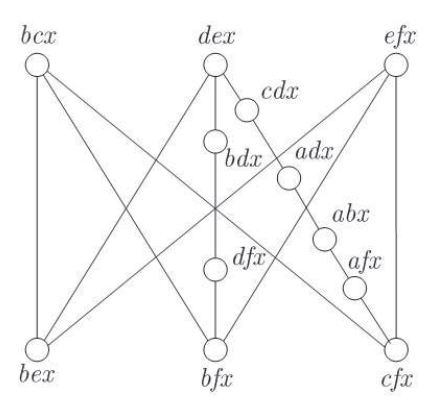

$(c)$

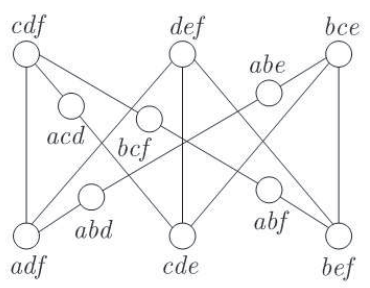

(b)

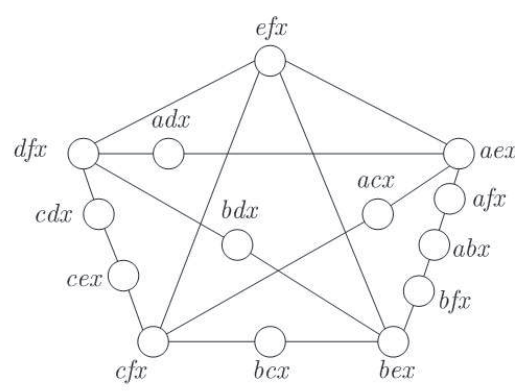

$(d)$

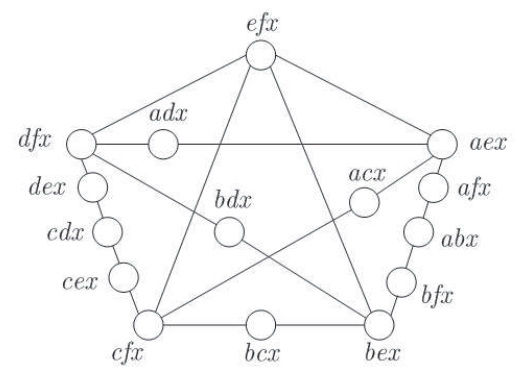

(e)

Figure 8: Some subgraph of $N_{i}$ for $i=1,5,11,16$ and 18

is planar.

Proof. The 3-jump graph $J_{3}\left(M_{i}\right)$ of a graph $M_{i}$ is shown in Figure 11 for $1 \leq i \leq 5$, and in Figure 12 for $6 \leq i \leq 11$. Thus, for each $i, J_{3}\left(M_{i}\right)$ is planar.

Next, we investigate that these graphs $M_{i}$, where $1 \leq i \leq 11$, of Figure 10 are maximal in the sense that its 3 -jump graph is planar. 

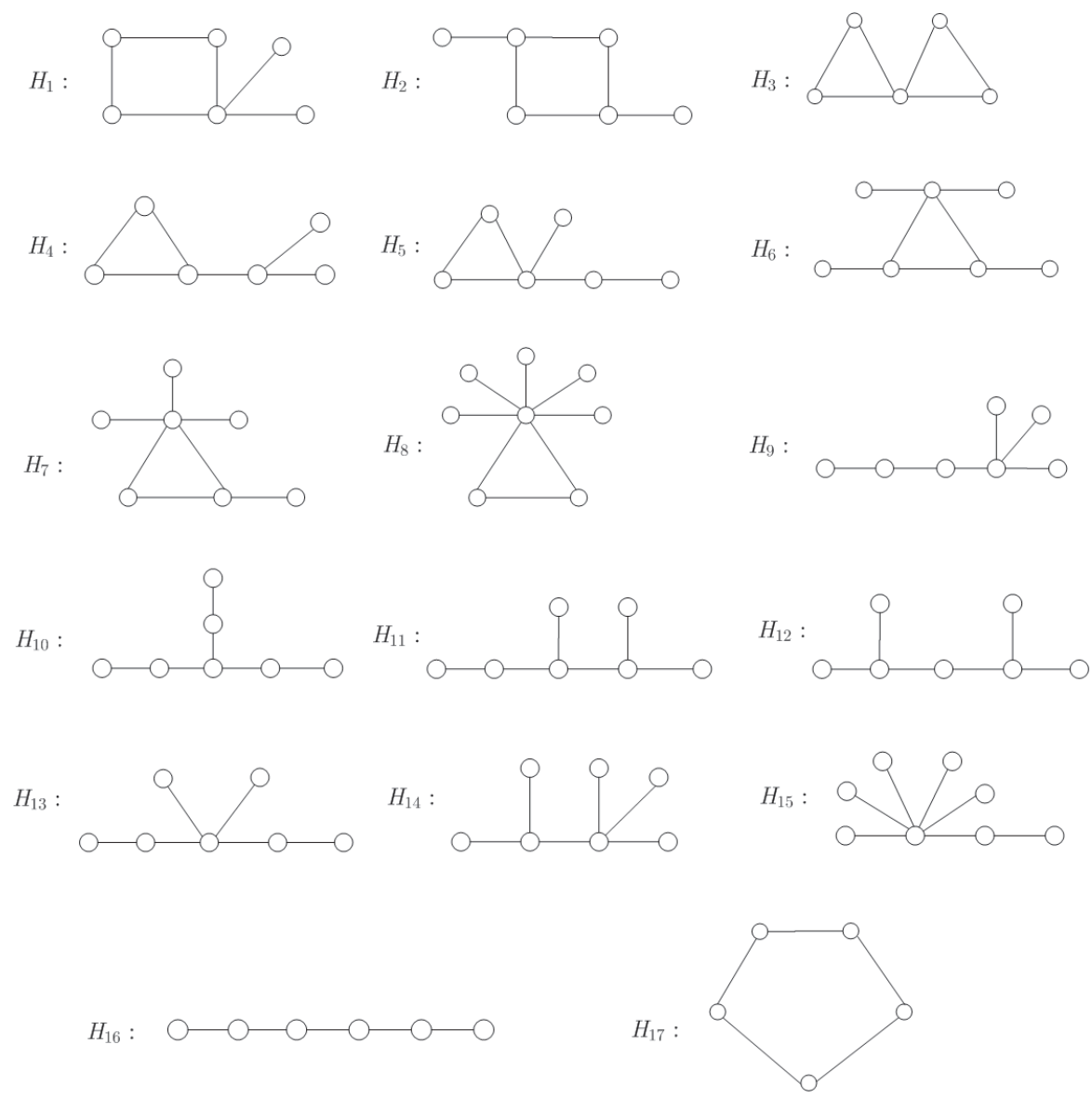

Figure 9: Graphs having a nonplanar 2-jump graph

Theorem 2.11. For a connected graph $G$ that is not a star, the 3-jump graph $J_{3}(G)$ is planar if and only if $G$ is a subgraph of $M_{i}$ for some $i$ where $1 \leq i \leq 11$ of Figure 10 .

Proof. It has been shown in Lemma 2.10 that, for $1 \leq i \leq 11, J_{3}\left(M_{i}\right)$ is planar. Thus if $G$ is a subgraph of $M_{i}$ for some $i$ where $1 \leq i \leq 11$ then $J_{3}(G)$ is planar by Lemma 2.3 .

For the converse, let $G$ be a connected graph that is not a star for whose $J_{3}(G)$ is planar. Then the connected graph $G$ may or may not contain cycles.

Case 1. $G$ is a tree. Since $G$ cannot contain $N_{20}=P_{6}$, it follows that $\operatorname{diam}(G) \leq 4$ and since $G$ is not a star, we have that $\operatorname{diam}(G) \geq 3$. Thus either $\operatorname{diam}(G)=3$ or $\operatorname{diam}(G)=4$. If $\operatorname{diam}(G)=3$ then $G$ is a double star. Observe 

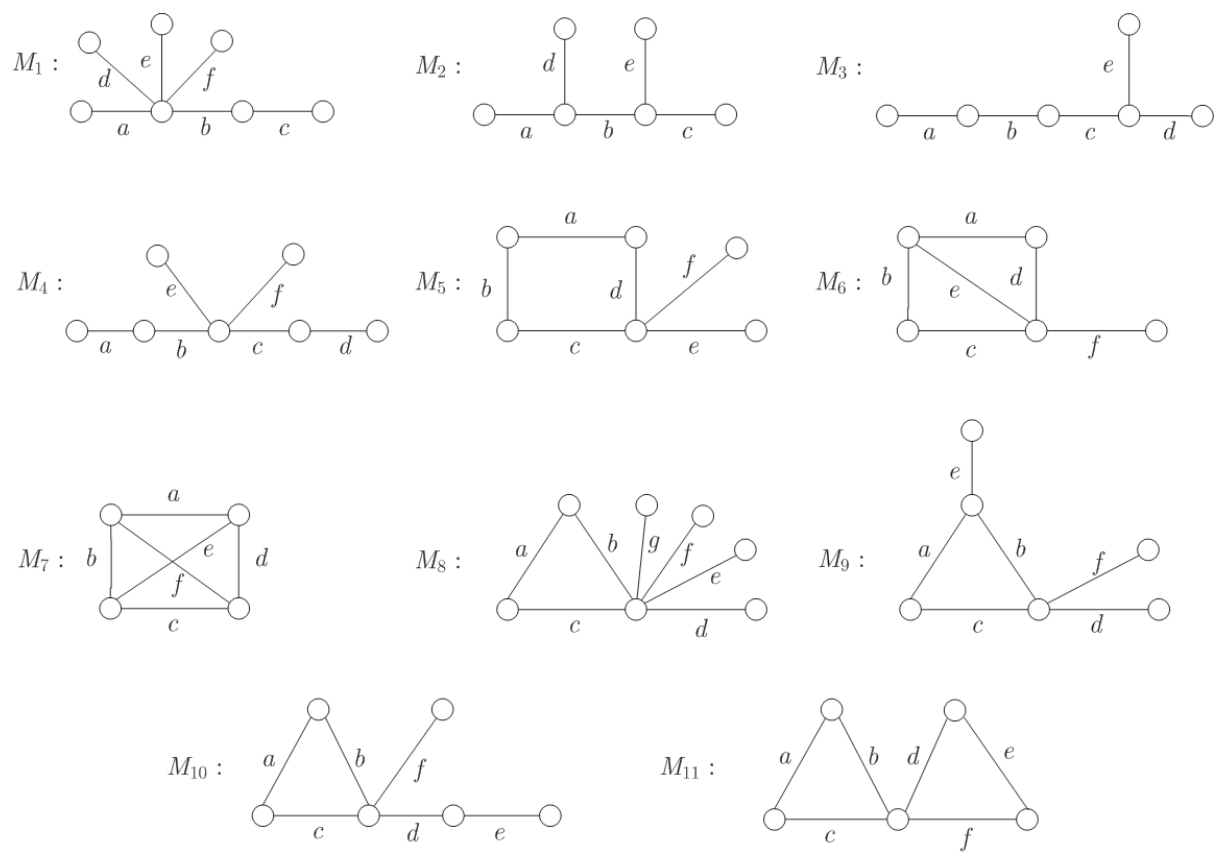

Figure 10: Graphs having a planar 3-jump graph

in this case that $G$ cannot contain $N_{1}$ and $N_{2}$, for otherwise $J_{3}(G)$ is nonplanar which is a contradiction. Thus $G$ is a subgraph of $M_{1}$ or $M_{2}$. (See Figure 13 for all graphs $G$ with $\operatorname{diam}(G)=3$ and $J_{3}(G)$ is planar.) Now, we assume that $\operatorname{diam}(G)=4$. Then $G$ cannot contain $N_{i}$, for $3 \leq i \leq 7$ as a subgraph. Therefore, $G$ is a subgraph of $M_{3}$ or $M_{4}$. (See Figure 14 for all graphs $G$ with $\operatorname{diam}(G)=4$ for whose $J_{3}(G)$ is planar.)

Case 2. $G$ contains cycles. Since $G$ cannot contain $N_{19}=C_{5}$, it follows that $G$ contains $C_{3}$ or $C_{4}$. We consider the following three subcases.

Subcase 2.1. $G$ contains $C_{4}$ but not $C_{3}$. Observe that $G$ cannot contain $N_{i}$ for each $i \in\{8,9,20\}$. Thus $G$ is a subgraph of $M_{5}$. (See Figure 15 for all graphs $G$ containing $C_{4}$ but not $C_{3}$ for whose $J_{3}(G)$ is planar.)

Subcase 2.2. $G$ contains both $C_{3}$ and $C_{4}$. Then, for $i \in\{10,11,19,20\}$, $N_{i}$ cannot be a subgraph of $G$. Therefore $G$ is a subgraph of $M_{6}$ or $M_{7}$. (See Figure 16 for all graphs $G$ containing $C_{4}$ and $C_{3}$ for whose $J_{3}(G)$ is planar.)

Subcase 2.3. $G$ contains $C_{3}$ but not $C_{4}$. Since the graph $G$ cannot contain $N_{i}$ where $12 \leq i \leq 18$ and $N_{20}, G$ is a subgraph of $M_{8}, M_{9}, M_{10}$ or $M_{11}$. (See Figure 17 for all graphs $G$ containing $C_{3}$ but not $C_{4}$ for whose $J_{3}(G)$ is planar.) 

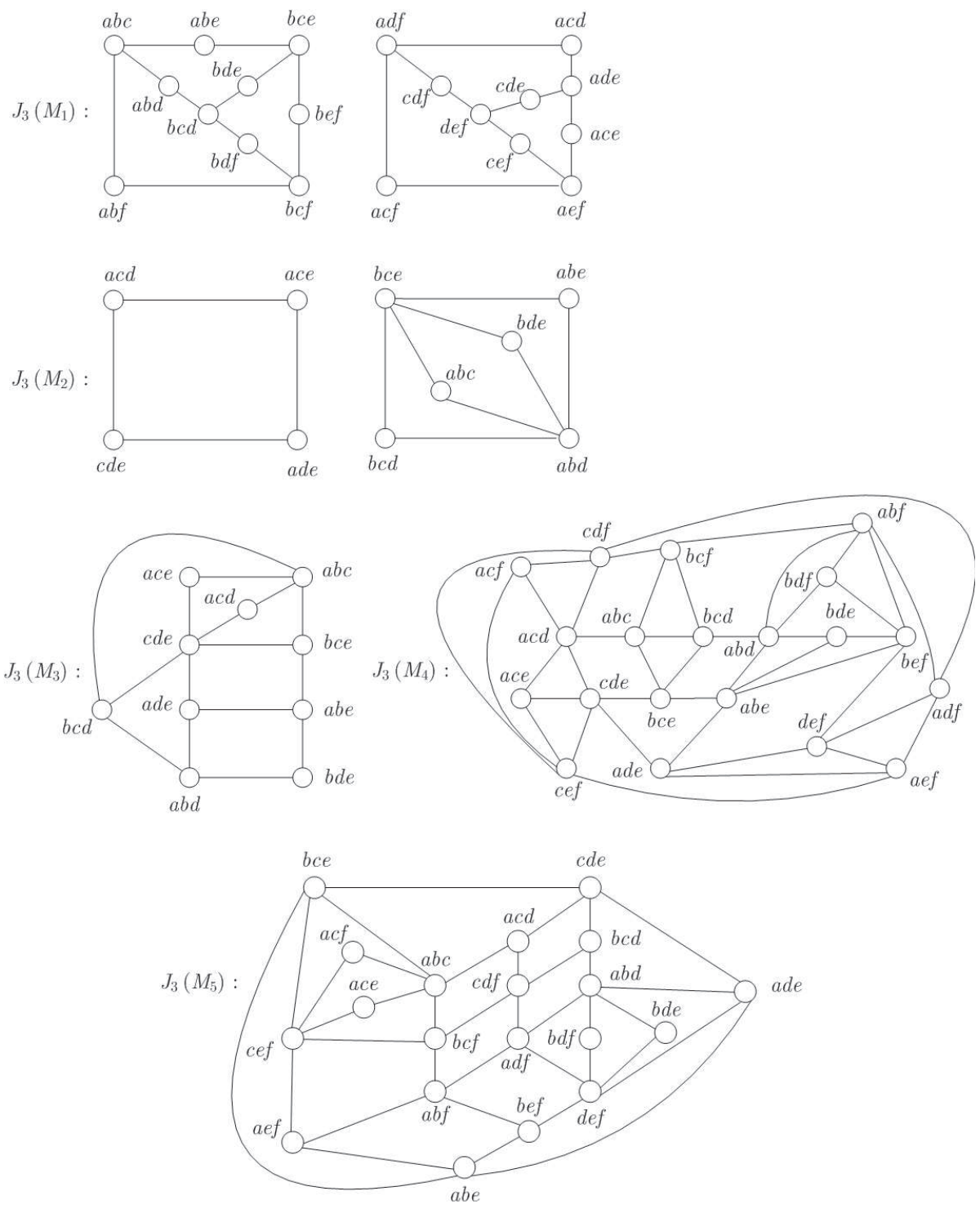

Figure 11: The 3-jump graphs of $M_{i}$ for $1 \leq i \leq 5$ 

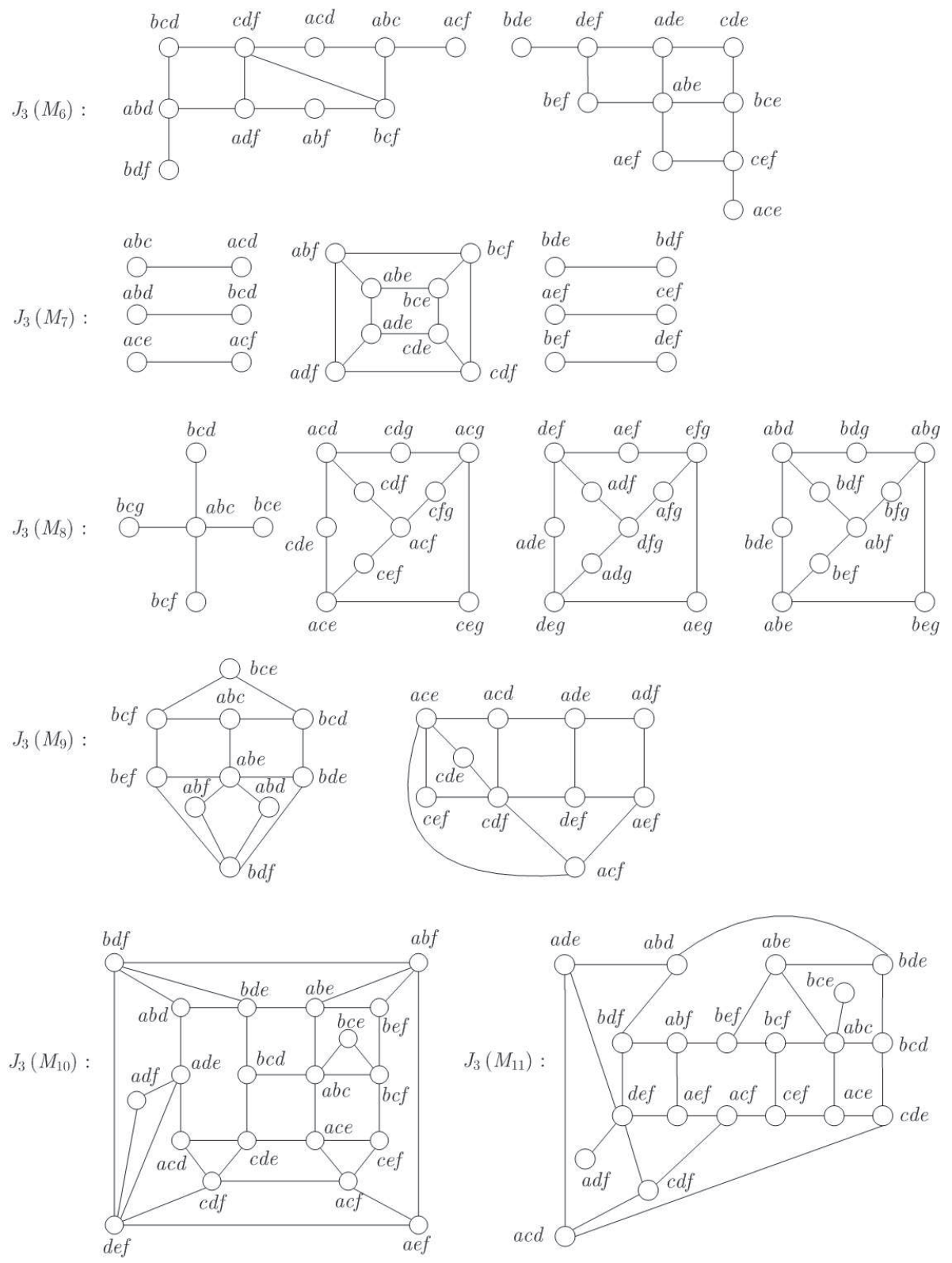

Figure 12: The 3-jump graphs of $M_{i}$ for $6 \leq i \leq 11$ 


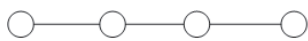

(a)

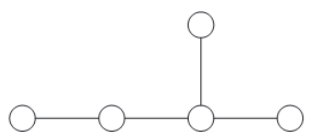

(b)

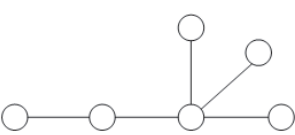

(c)

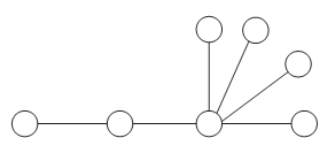

(d)

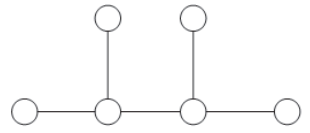

(e)

Figure 13: All trees with diameter 3 for whose the 3 -jump graph is planar

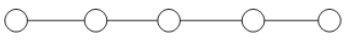

(a)

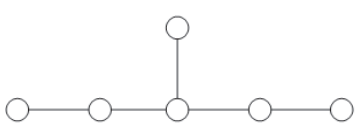

(c)

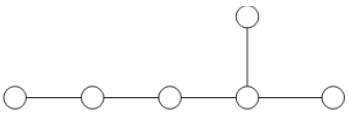

(b)

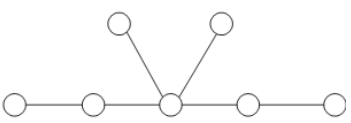

(d)

Figure 14: All trees with diameter 4 for whose the 3 -jump graph is planar

We next present another characterization of graphs for whose the 3-jump graph is planar and also show that these graphs $N_{i}$, for $1 \leq i \leq 20$ are minimal according to its 3 -jump graph being nonplanar.

Corollary 2.12. For a connected graph $G$ that is not a star, the 3-jump graph $J_{3}(G)$ is planar if and only if $G$ does not contain any of $N_{i}$ for $1 \leq i \leq 20$ of Figure 7 as a subgraph.

Proof. If $G$ contains $N_{i}$ for some $i$ where $1 \leq i \leq 20$, then $J_{3}(G)$ contains $J_{3}\left(N_{i}\right)$, by Lemma 2.3, and so $J_{3}(G)$ is nonplanar since $J_{3}\left(N_{i}\right)$ is nonplanar.

For the converse, we assume that $G$ does not contain any of $N_{i}$ for $1 \leq i \leq$ 20. We consider two cases.

Case 1. $G$ is a tree. Since $G$ does not contain $N_{20}=P_{6}$, it follows that $\operatorname{diam}(G) \leq 4$ and since $G$ is not a star, we have that $\operatorname{diam}(G) \geq 3$. Thus either 


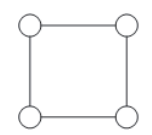

(a)

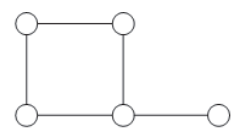

(b)

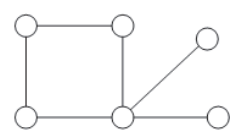

(c)

Figure 15: All graphs that contain $C_{4}$ but not $C_{3}$ for whose the 3 -jump graph is planar

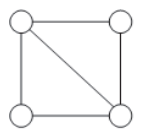

(a)

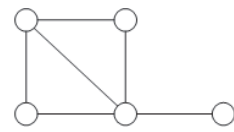

(b)

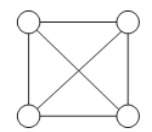

(c)

Figure 16: All graphs that contain $C_{3}$ and $C_{4}$ for whose the 3 -jump graph is planar

$\operatorname{diam}(G)=3$ or $\operatorname{diam}(G)=4$. If $\operatorname{diam}(G)=3$ then since $G$ does not contain $N_{1}$ and $N_{2}$, we have that $G$ is a subgraph of $M_{1}$ or $M_{2}$. Thus $J_{3}(G)$ is planar. Now, if $\operatorname{diam}(G)=4$, then again since $G$ does not contain $N_{i}$, where $3 \leq i \leq 7$ as a subgraph, $G$ is a subgraph of $M_{3}$ or $M_{4}$. Therefore $J_{3}(G)$ is planar.

Case 2. $G$ contains cycles. Since $G$ does not contain $N_{19}=C_{5}$, it follows that $G$ contains $C_{3}$ or $C_{4}$. If $G$ contains $C_{4}$ but not $C_{3}$ then since $G$ does not contain $N_{i}$ for $i \in\{8,9,20\}, G$ is a subgraph of $M_{5}$ and so $J_{3}(G)$ is planar. Next, if $G$ contains both $C_{3}$ and $C_{4}$ then since none of $N_{i}$ for $i \in\{10,11,19,20\}$ is contained in $G, G$ is a subgraph of $M_{6}$ or $M_{7}$ and thus $J_{3}(G)$ is planar. Finally, if $G$ contains $C_{3}$ but not $C_{4}$ then since $G$ does not contain $N_{i}$ where $12 \leq i \leq 18$ and $N_{20}, G$ is a subgraph of $M_{i}$ for some $8 \leq i \leq 11$ and thus $J_{3}(G)$ is planar.

\section{Final Remarks}

In this paper, we have characterized connected graphs whose the 3-jump graph is planar. A natural question arises what the characterization of a connected graph whose the $k$-jump graph where $4 \leq k \leq m-4$ is planar. 


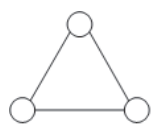

(a)

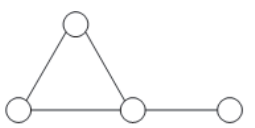

(b)

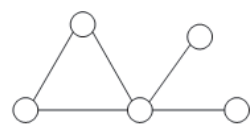

(c)

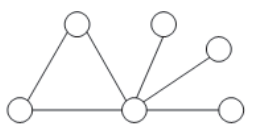

(d)

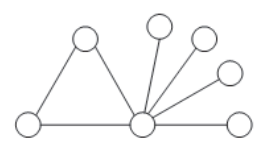

(e)

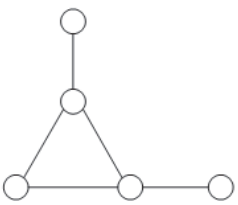

(h)

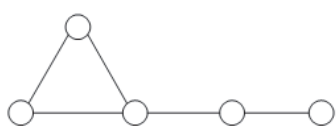

(f)

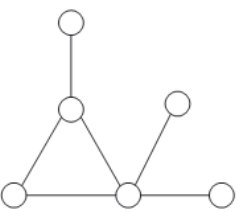

(i)

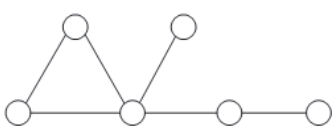

(g)

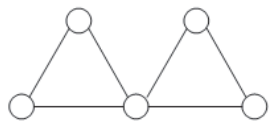

(j)

Figure 17: All graphs that contain $C_{3}$ but not $C_{4}$ for whose the 3 -jump graph is planar

\section{Acknowledgments}

Research is supported by Faculty of Science, Srinakharinwirot University, Year 2015.

\section{References}

[1] G. Chartrand, H. Hevia, E.B. Jarrett and M. Schultz, Subgraph distance in graphs defined by edge tranfers, Discrete Math., 170 (1997), 63-79.

[2] G. Chartrand, L. Lesniak and P. Zhang, Graphs \& Digraphs: 5th Edition, Chapman \& Hall/CRC, USA (2010).

[3] H. Hevia, D.W. VanderJagt, and P. Zhang, On the planarity of jump graphs, Discrete Math., 220 (2000), 119-129.

[4] K. Kuratowski, Sur le probléme des courbes gauches en topologie, Fund. Math., 15 (1930), 270-283. 